\title{
Lp (A) and Aortic Stenosis
}

\section{Scott W Shurmur*}

Department of Internal Medicine, University of Texas Tech Health Sciences Center, USA

*Corresponding author: Scott W Shurmur, Department of Internal Medicine, University of Texas Tech Health Sciences Center, USA.
Received Date: April 20, 2020

Published Date: April 27, 2020

\section{Case Report}

Lipoprotein (a), commonly called Lp "little a", is composed of an LDL-like particle, with an apolipoprotein B component covalently bound to apo (a). The structure of the apo (a) portion is somewhat similar to plasminogen, though number and repetition of the "Kringle" portions differs [1]. The atherogenicity of Lp (a) is increasingly appreciated. Recent genetic study confirms its strong association with clinical atherosclerosis. Additionally, some iso form of Lp (a) are strongly associated with calcific aortic stenosis (Figure 1).

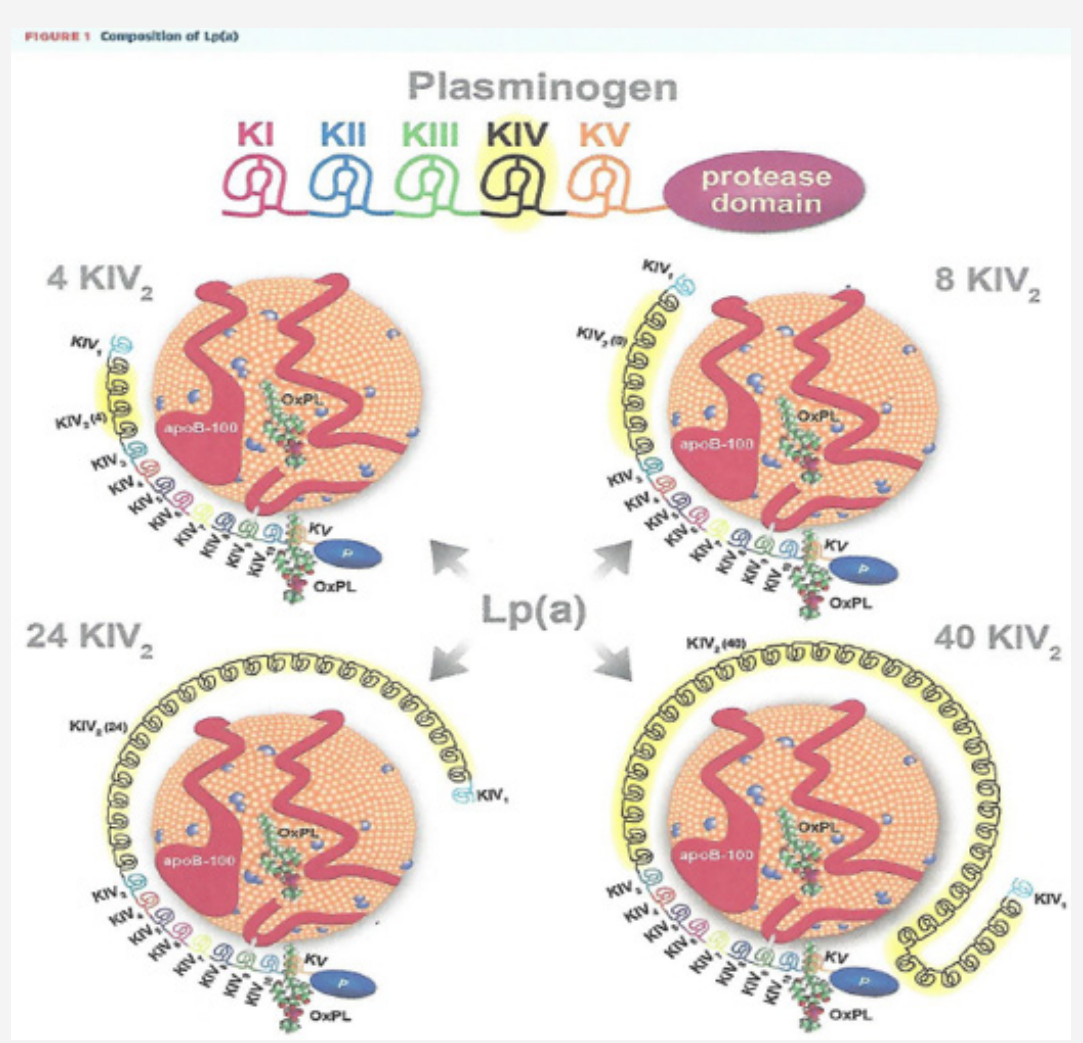

Figure 1: Lipoprotein [ $\mathrm{Lp}(\mathrm{a})]$ is composed of apolipoprotein B-100 (apoB-100) covalently bound to apolipoprotein (a) [apo(a)], which is derived from Kringle IV (KIV) and KV, and the protease domain of plasminogen has 1 copy each of KI to KV and an active protease domain. Apo(a) contains 10 subtypes of KIV repeats, composed of 1 copy each of $\mathrm{KIV}_{1}$, multiple copies of $\mathrm{KIV}_{2}$, and 1 copy of $\mathrm{KIV}_{3} \ldots . .10_{v} \mathrm{KV}$ and an inactive protease-like (p) domain. In these examples, apo(a) isoforms of 4, 8, 24 and 40 KIV2 repeats are shown, representing 13, 17, 33 and 49 total KIV repeats. Oxidized phospholipids (OXPL), represented there by 1-palmicoyl-2-oxovaleray-sn-glycero-3-phosphocholine (POVPC), are present covalently bound to apo(a), and also dissolved in the lipid phase of apoB-100. 
Specifically, SNP rs 10455872 is strongly associated with markedly elevated Lp (a) levels (greater than $50 \mathrm{mg} / \mathrm{dl}$ ) and the only monogenetic risk factor linked to calcific aortic valve stenosis in multiple racial groups. Mechanistically, autotaxin, which is involved in the lysophosphatidylcholine pathway, appears to be a promoter of inflammation, fibrosis and cell motility [2]. Several clinical trials have shown an association of elevated Lp (a) levels, and increased rate of progression of calcific aortic stenosis. Therapies targeting Lp (a) are in development, including highly specific antisense oligonucleotides [1].

\section{Acknowledgement}

None.

\section{Conflict of Interest}

No conflict of interest.

\section{References}

1. Tsimikas S (2017) A Test in Context: Lipoprotein(a): Diagnosis, Prognosis, Controversies, and Emerging Therapies. J Am Coll Cardiol 69(6): 692-711.

2. Bouchareb R, Mahmut A, Nsaibia MJ, Boulanger MC, Dahou A, et al. (2015) Autotaxin Derived From Lipoprotein(a) and Valve Interstitial Cells Promotes Inflammation and Mineralization of the Aortic Valve. Circulation 132(8): 677-690. 